\title{
Modelo Batimétrico derivado de imágenes Landsat ETM+ en zonas de arrecifes tropicales
}

\author{
Alexander Ariza* \\ Héctor Mauricio Ramírez**
}

Recibido el 29 de julio de 2014; aceptado el 6 de agosto de 2014

\begin{abstract}
For mapping and monitoring coral reefs, a bathymetric map is useful as a base map. Different methods have already been developed to map bathymetry using remote sensing. Two main groups can be distinguished. One group is using active remote sensing data; the other is based on passive sensor generated multi-spectral information. In this article, the focus is on the passive data method. A modified DOP "depth of penetration" mapping method was implemented on a Landsat ETM+ image over Archipélago San Andrés (Colombia). Depth measurements used in the validation of the model were derived from the interpolation of bathymetric maps. The accuracy test revealed that the resulting bathymetric map is useful for coral reef mapping, but care should be taken when using it. Deviations from reality were caused by assumptions inherent to the theory used, data interpolation, satellite image characteristics and errors during implementation of the method.

Key words: coral reef, remote sensing, bathymetry, Landsat ETM+, Caribbean Sea.

\section{Resumo}

Para a cartografia e monitoramento dos arrecifes de corais, um mapa batimétrico é tão útil como um mapa básico. Diferentes métodos têm sido desenvolvidos para

* $\mathrm{PhD}(\mathrm{c})$ en Cartografía, SIG y Teledetección de la Universidad de Alcalá de Henares, Madrid. Grupo de Percepción Remota del Centro de Investigación y Desarrollo en Información Geográfica [CIAF, Instituto Geográfico Agustín Codazzi (IGAC)], correo electrónico: alexanderariza@igac.gov.co

** M.Sc. en Magister en Geografía de la Universidad Pedagógica y Tecnológica de Colombia. Grupo de Percepción Remota del Centro de Investigación y Desarrollo en Información Geográfica [CIAF, Instituto Geográfico Agustín Codazzi (IGAC)], correo electrónico: hmramirez@igac.gov.co
\end{abstract}


cartografar a batimetria usando-se sensores remotos. Dois grandes grupos podem se distinguir. O primeiro utiliza dados de sensoriamento remoto de sensores ativos e, o segundo se baseia no emprego de sensores passivos para gerar informação multiespectral. Este artigo se concentra no método de dados provenientes de sensores passivos. Trata-se de uma modificação ao método DOP (profundidade de penetração) mediante espectrometria que se levou a cabo em imagens Landsat ETM+ sobre o Arquipélago de San Andrés e Província (Colômbia). As medições de profundidade utilizadas na validação do modelo foram derivadas de leitura em campo com uma ecosonda e interpolação de mapas batimétricos. A exatitude da prova revelou que o modelo batimétrico resultante é útil para o mapeamento em zonas de arrecifes de coral no Mar do Caribe em profundidades de até $25 \mathrm{~m}$, aproximadamente. Os dados não condizentes com a realidade foram gerados por superposições inerentes a teoria utilizada, a interpolação de dados, as características da imagem de satélite e, os erros durante a execução do método.

Palavras chave: arrecifes de coral, sensores remotos, batimetria, Landsat ETM+, Mar do Caribe.

\section{Resumen}

Para la cartografía y monitoreo de los arrecifes de coral, un mapa batimétrico es útil como un mapa base. Diferentes métodos han sido desarrollados para cartografiar la batimetría usando sensores remotos. Dos grandes grupos se pueden distinguir. El primero utiliza datos de teledetección de sensores activos y el segundo se basa en el empleo de sensores pasivos para generar información multiespectral. Este artículo se centra en el método de datos provenientes de sensores pasivos. Una modificación al método DOP "Profundidad de penetración" mediante espectrometría que se llevó a cabo en imágenes Landsat ETM+ sobre Archipiélago de San Andrés y Providencia (Colombia). Las mediciones de profundidad utilizadas en la validación del modelo fueron derivadas de lecturas de ecosonda en campo e interpolación de mapas batimétricos. La exactitud de la prueba reveló que el modelo batimétrico resultante es útil para el mapeo en zonas de arrecifes de coral del mar Caribe hasta $25 \mathrm{~m}$ de profundidad aproximadamente. Los datos no conformes a la realidad fueron generados por suposiciones inherentes a la teoría utilizada, la interpolación de datos, las características de la imagen de satélite y los errores durante la ejecución del método.

Palabras clave: arrecifes de coral, sensores remotos, batimetría, Landsat ETM+, mar Caribe.

\section{Introducción}

Los sensores remotos proveen una herramienta efectiva en la observación y monitoreo de arrecifes coralinos alrededor del mundo, permitiendo la caracterización de su 
estructura interna y externa de los diferentes hábitats, mostrando una aproximación de la complejidad de los ecosistemas de arrecife.

El empleo y procesamiento de imágenes Landsat permite la discriminación de la complejidad presente en más de 13 clases diferentes de corales (Serge Andréfouët, 2003). Los mapas de batimetría son de gran utilidad para el manejo y gestión de zonas protegidas, así como para la evaluación de riesgo en ambientes marinos (Clark, 1997). La información de batimetría actual es muy pobre, desactualizada o a menudo inexistente, sobre todo en países en vía de desarrollo.

Debido a la poca profundidad y/o ubicación en el océano, no siempre es adecuado realizar campañas de batimetría por métodos convencionales como un ecosondeo. De este modo las nuevas técnicas de percepción remota se presentan como una alternativa metodológica viable para generar información batimétrica confiable.

Existen diferentes métodos de percepción remota en la obtención de mapas de batimetría, los cuales se pueden agrupar en dos grandes grupos. Aquellos derivados de la utilización de datos de sensores activos (Lidar batimétrico) y los basados en la información multiespectral de sensores pasivos (Imágenes de ópticas), como en el caso del presente artículo. Un ejemplo de este último es el uso de modelos de transferencia radiativa y análisis multitemporal de imágenes Landsat, dichos modelos permiten la discriminación de los límites en los arrecifes de coral con una aproximación de hasta 17 metros de profundidad (Hiroya Yamano, 2004).

La teoría basada en el modelo de reflexión de fondo (Ji, Civco y Kennard, 1992) establece que hasta una cierta profundidad parte de la señal registrada por el sensor es reflejada por el fondo. En casos de zonas de aguas claras (Jerlov, 1976), el agua en el fondo del mar puede reflejar la luz suficiente para ser detectada por un sensor del satélite hasta una profundidad de 30 metros (Green, Gmumby y Edwards, 2000).

Otros estudios evaluaron diferentes técnicas de datos de sensores pasivos (Benny y Dawson, 1983), basados en la correlación entre la profundidad calculada mediante los diferentes métodos y la profundidad real en un área de control. Dichos estudios llegaron a la conclusión de que el método DOP-mapping (Deep of penetration) o "Profundidad de penetración" desarrollado por Jupp (1988) fue el más exacto. En nuestro caso este estudio se centra en el empleo del método DOP, modificado por Green et al. (2000) y Vanderstraete, Goossens y Ghabour (2003) y su modificación a través de la respuesta espectral de la columna de agua medida en campo.

El principio básico del método de Jupp (1988) consiste en que las diferentes longitudes de onda del espectro electromagnético penetran en el agua en mayor o menor medida. La luz de mayor longitud de onda en la parte visible del espectro (banda roja) tiene un alto coeficiente de atenuación, mucho más que en longitudes de onda corta, por ejemplo en la región del azul. A una profundidad determinada, la profundidad máxima de penetración para la región roja del espectro, detectada en la 
banda 3 del sensor Landsat 7 ETM+ $(0.63-0.69 \mu \mathrm{m})$, ha sido totalmente atenuada. Sin embargo, a esta profundidad todavía habrá un poco de luz detectable por bandas 2 (verde) y 1 (azul) en el sensor ETM+.

Las zonas DOP están delimitadas por el valor máximo de profundidad de penetración de las bandas en longitudes de onda más cortas (Green, Gmumby y Edwards, 2000). Tres supuestos adicionales son base en la construcción del método DOP (Green, Gmumby y Edwards, 2000): la atenuación de la luz es una función exponencial de la profundidad, la calidad del agua y el sustrato (dichos valores no varían dentro de la imagen).

\section{Área de estudio}

Como área de estudio se tomaron los arrecifes de coral del archipiélago de San Andrés y Providencia, Colombia $\left(12^{\circ} 35^{\prime} \mathrm{N}, 81^{\circ} 40^{\prime} \mathrm{O}\right)$, situados en la parte suroeste del mar Caribe. Y como área de prueba se seleccionó el arrecife de coral perteneciente a la isla de San Andrés (Figura 1). El territorio del archipiélago se encuentra en un entorno medioambiental único, conformado por un conjunto de islas, cayos e islotes que posee una extensión total de $350,000 \mathrm{~km}^{2}$.

La situación en el mar Caribe al igual que en el archipiélago de San Andrés y Providencia es especialmente preocupante, debido a un desarrollo costero incontrolado y a la presión que el turismo ejerce; afectando numerosas estructuras de arrecifes y promoviendo un peligro potencial en más áreas futuras (Mow, 2005).

\section{Metodología}

\section{Datos de campo}

Esta metodología incluye la obtención de datos de campo mediante un muestreo aleatorio estratificado de puntos de verificación. Las observaciones se realizaron en el mar Caribe, mediante un equipo de georreferenciación (GPS) en un sistema de coordenadas UTM para X, Y y Z sobre el elipsoide WGS84 con un error medio cuadrático RMS no mayor a $1 \mathrm{~m}$. Los valores de profundidad $(Z)$ utilizados en el proceso de validación del modelo DOP se obtuvieron bajo el sistema monohaz, mediante una ecosonda Odom Hydrotrac en un sistema de posicionamiento GPS Diferencial DSM 132 TRIMBLE complementado con el paquete hidrográfico HYPACK MAX con una precisión de entre 0.3-1.0m. Para ello se empleó una embarcación hidrográfica de tipo Zodiac con el fin de alcanzar las áreas de poca profundidad (Figura 2).

En este estudio las mediciones de espectrometría fueron tomadas mediante un espectroradiómetro HR4000 de Oceans Optics, con el cual se obtuvieron las firmas espectrales utilizadas en el proceso de cálculo del coeficiente de atenuación y corrección de la columna de agua. Esta toma de datos se realizó a través del buque 


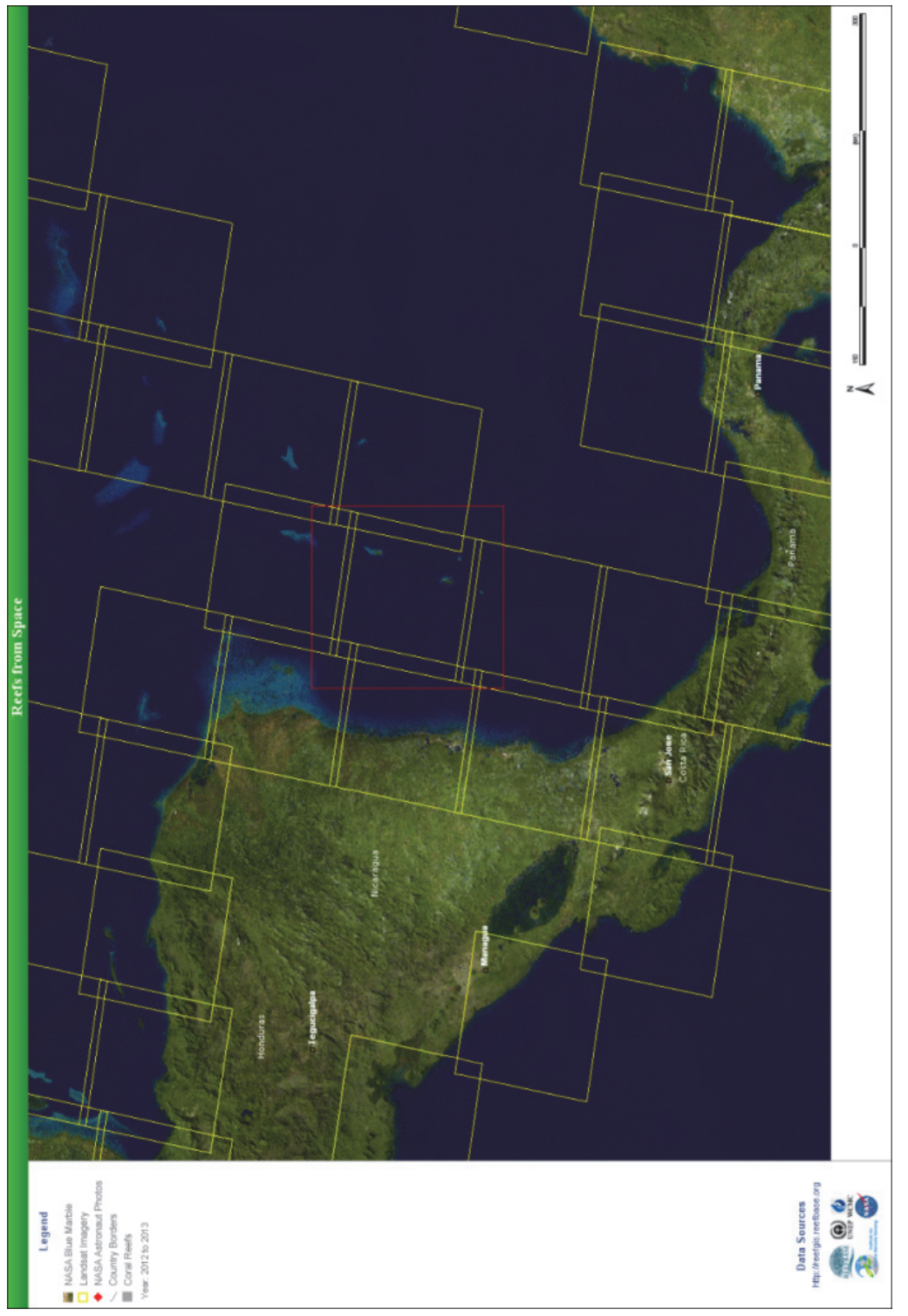

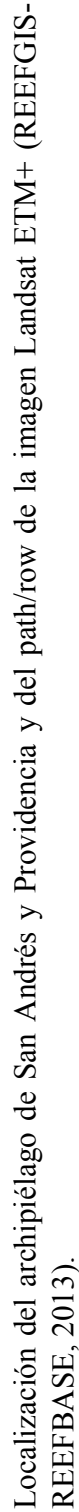

تِّ 
oceanográfico "ARC Providencia" del Centro de Investigaciones Oceanográficas e Hidrográficas ( $\mathrm{CIOH}$ ) adscrito a de la Dirección General Marítima (DIMAR) (Figura 2).

Los datos capturados fueron posprocesados en laboratorio con el fin de validar y correlacionar el modelo DOP, a partir de los valores batimétricos obtenidos en campo contra los datos derivados de la imagen del satélite Landsat ETM+ y de sus valores de radiancia ( $\mathrm{Li}$ ) máxima, media y mínima entre las bandas multiespectrales (1-4).

\section{Datos de marea}

Los promedios de marea diaria en el archipiélago de San Andrés son de $0.6 \mathrm{~m}$ aproximadamente. Esto se ve aumentado por un efecto de evaporación estacional, un estimado de $0.5 \mathrm{~m}$ entre la época de verano y el invierno (Edwards, 1987). Es importante compensar el efecto de la marea en la imagen procesada y durante el muestreo de datos para convertir un mapa batimétrico en datum vertical. Los datos y previsiones de mareas se obtuvieron a partir de los parámetros llamados constituyentes armónicos, que están basados en los históricos de mediciones de las mareas (U.S. Government Funding, 1994). Para ello se utiliza el programa de predicción XTide (Harmonic tide clock and tide predictor) Versión 2.0, disponible en $<$ http://www.flaterco.com/xtide/>.

Se ha extrapolado el uso de estos datos de marea en función del método inverso de la distancia ponderada (IDW). La marea calculada en el momento de la adquisición de la imagen (26 de julio de 2001, 08:29 GMT) fue de 0.24m por encima del punto de referencia (Vanderstraete, Goossens y Ghabour, 2003).

\section{Datos de satélite}

Se adquirió una imagen Landsat ETM+ a nivel 2 (Ortorrectificada) - path/row: 014/051 (Figura 2), que data del 26 de julio de 2001. Ésta se utilizó para determinar la profundidad de la zona sublitoral del área de estudio. Las bandas 1, 2 y 3 fueron utilizadas debido a que estas longitudes de onda no son totalmente absorbidas por la columna de agua (Vanderstraete, Goossens y Ghabour, 2003). De igual manera, la banda 4 participó en la cartografía batimétrica de la zona intermareal.

El procesamiento de imágenes se realizó mediante el software Geomática PCI 2013, utilizado para georreferenciar las capas batimétricas auxiliares en el área de estudio. Se tomaron 21 puntos de chequeo (Check point), de un total de 63 puntos de control GCP, obteniendo un error $(\mathrm{RMS}=0.406)$, dentro del límite aceptable de 0.5 píxeles. La georreferenciación se basó en un sistema específico de coordenadas (UTM - WGS84 - Zona 17N). La imagen de satélite fue corregida atmosférica y radiométricamente, con el fin de obtener los valores físicos de la reflectividad del fondo de arrecife. 


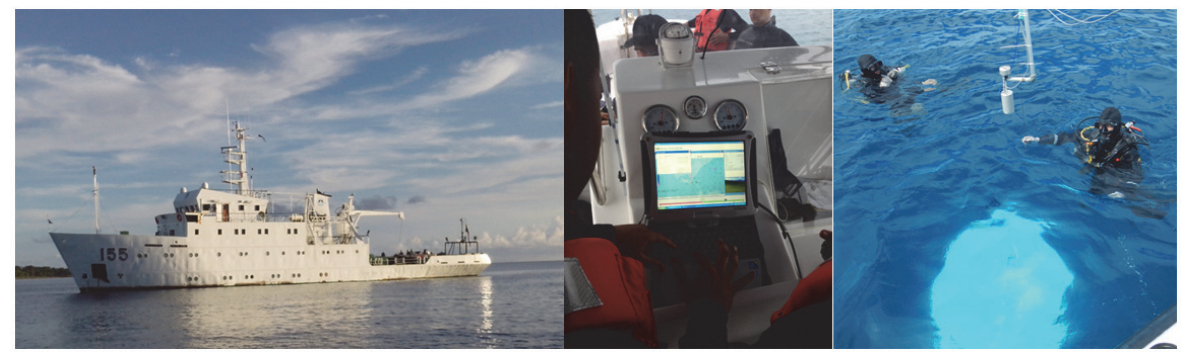

Figura 2. Buque oceanográfico "ARC Providencia" a la izquierda, Ecosonda Monohaz en el centro y mediciones con espectrorradiómetro HR4000 a la derecha (CIAFDIMAR, 2013).

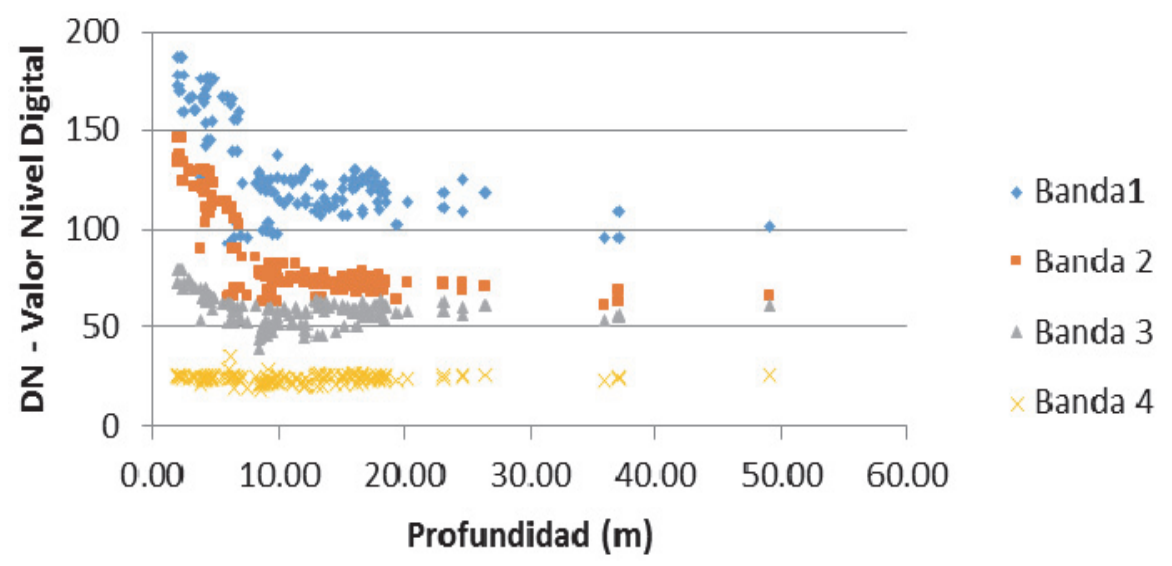

Figura 3. Relación entre los valores de ND y profundidad (estudio propio, 2013).

\section{Corrección de imágenes}

Este proceso se desarrolló en dos partes. En primer lugar, se convirtieron los niveles digitales (DN) a valores de radiancia (Li). Posteriormente, se convirtieron estos valores de radiancia a valores de reflectancia (Chander et al., 2009).

Para convertir los DN a radiación, se utilizaron los datos de calibración de la escena disponibles en el archivo de cabecera. Se emplearon los valores de ganancia y polarización (o desplazamiento).

La fórmula para convertir los $\mathrm{DN}$ a radiancia es la siguiente:

$$
L \lambda=\text { gain } * D N+\text { bias }
$$


Donde:

$L \lambda$ es el valor de radiancia del píxel

$D N$ es el valor digital del píxel

Gain es el valor de la ganancia en la banda

Bias es el valor de la polarización en la banda

Una vez obtenidos los valores de radiancia, éstos son convertidos a reflectancia mediante la siguiente fórmula:

$$
\rho \lambda=\frac{(\pi * L \lambda * d 2)}{E A S U N \lambda * \operatorname{Cos} \theta_{s}}
$$

Donde:

$P \lambda=$ reflectancia en unidades planetarias

$L \lambda=$ Radiancia espectral

$d 2=$ Distancia entre el Sol y la Tierra en unidades astronómicas

$E S U N \lambda=$ Irradiancia solar media estratosférica

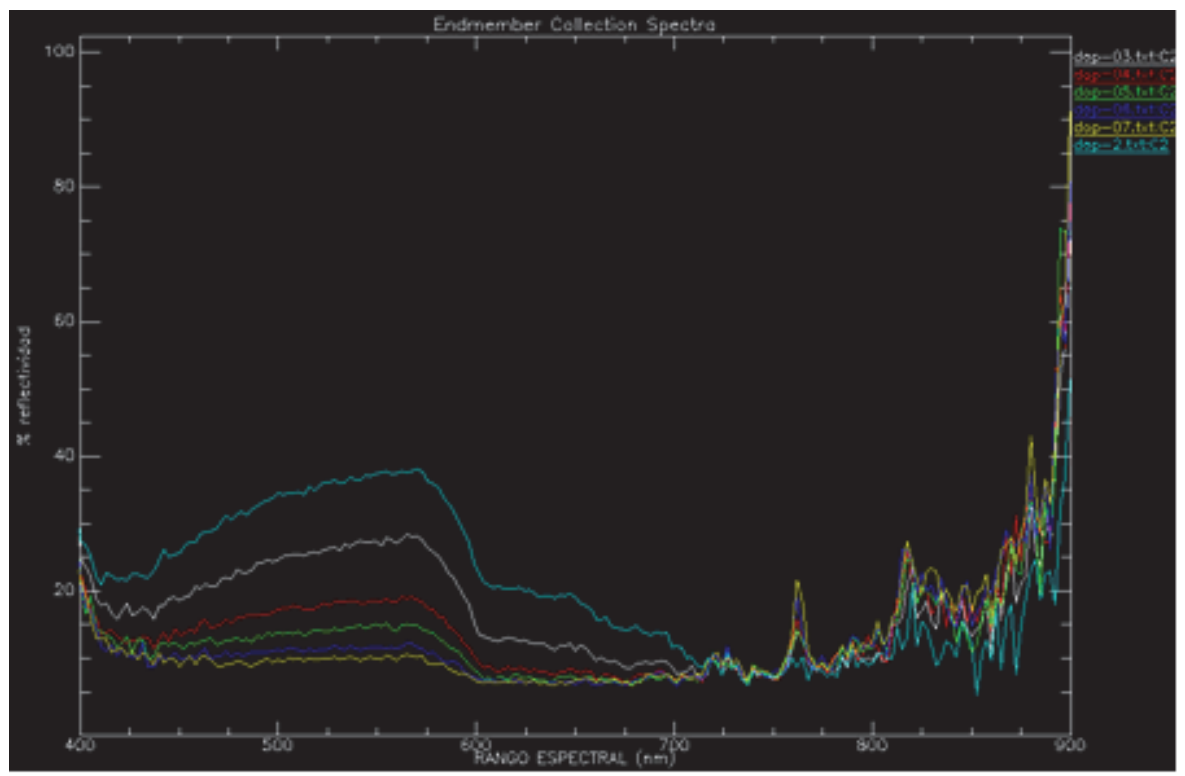

Figura 4. Firmas espectrales obtenidas en las zonas de DOP para la determinación del coeficiente de atenuación a diferentes profundidades (estudio propio, 2013). 


\section{Determinación de las zonas DOP}

La imagen utilizada se ha dividido en cuatro diferentes zonas DOP (Green, Edwards y Mumby, 2000). Se define una zona de DOP como "una región en la que el espectro se refleja en una banda, pero no en el siguiente". Por ejemplo, la primera zona de DOP-1 representa aquellas profundidades en la imagen en la que sólo la banda 1 del sensor ETM+ recibe la reflectancia de la parte inferior. La zona DOP-2 contiene la reflexión del azul y verde, pero no la del rojo e infrarrojo cercano, y así sucesivamente.

En primer lugar, hay que identificar la reflexión en el área de agua profunda (Liœ). Estos valores de niveles digitales DN en aguas profundas fueron determinados en un subconjunto de la imagen a través de una zona con una profundidad mayor a $50 \mathrm{~m}$, como está normalmente señalado en una carta náutica. Se parte de la suposición de que en aguas profundas la señal recibida en el sensor está compuesta en su totalidad por la reflexión de la atmósfera, la superficie del agua y la columna de agua (Green, Edwards y Mumby, 2000); por lo tanto, la media de reflectancia en aguas profundas (Liœmedia) se utilizó para corregir más o menos los efectos de la atmósfera y de la columna de agua en la señal (Tabla 1). Por otra parte, el máximo de reflectancia de aguas profundas (Liøomáximo), en cada banda se utiliza para delinear las diferentes zonas DOP (Tabla 3). Si el valor del DN en una banda de i es mayor que Liœ max, se supone que parte de la reflectancia del fondo marino está presente en la señal recibida por el sensor (Green, Edwards y Mumby, 2000).

En segundo lugar, se calculó la profundidad máxima de penetración en metros de las bandas 1 a la 4 para el sensor ETM+ Landsat 7 (Tabla 2). Para cada banda i, esta profundidad fue determinada por la mayor profundidad de los píxeles con mayor valor de DN que el valor máximo de aguas profundas (Liœmax) (Edwards, A., 1999).

Para estimar la máxima profundidad de penetración para cada ancho de banda se utilizaron 200 puntos de observación; estos puntos fueron tomados en un intervalo homogéneo en los mapas y cartas batimétricas. Los valores observados de profundidad de referencia fueron corregidos con los datos de marea correspondientes a la fecha de adquisición de la imagen. Estos puntos se superponen a la imagen de satélite georreferenciadas, obteniendo el valor de DN y profundidad para cada banda (Figura 3).

\section{Modificación al método DOP (Profundidad de penetración)}

Una descripción detallada del procedimiento del método DOP "Profundidad de penetración" está descrito en el manual de Bilko para Windows [Edwards A. (1999) y Green, Edwards y Mumby (2000)]. Sin embargo, el procedimiento se puede dividir en dos secciones. En primer lugar, se determinan las cuatro diferentes zonas DOP y, en segundo lugar, la profundidad calculada para cada píxel de la imagen en cada zona. 


\section{Tabla 1}

Valores máximos, medios y mínimos de los DN en las bandas 1 a la 4 de la imagen ETM+ sobre el área de agua profunda

\begin{tabular}{lcccc}
\hline \multicolumn{5}{c}{ LANDSAT 7 ETM+ } \\
(Path/row: 014/051; date: 26/07/2001) & & \\
\hline & Banda 1 & Banda 2 & Banda 3 & Banda 4 \\
\hline Maximum DN-value for deep water (Liøomax) & 230 & 200 & 130 & 24 \\
Minimum DN-value for deep water (Liømin) & 90 & 67 & 62 & 21 \\
Mean DN-value for deep water (Liømean) & 160 & 134 & 96 & 23 \\
\hline
\end{tabular}

Fuente: CIAF-DIMAR (estudio propio, 2013).

Tabla 2

Valores máximos de profundidad de penetración (DOP) en las bandas 1 a la 4

\begin{tabular}{|c|c|c|c|c|}
\hline \multicolumn{5}{|c|}{$\begin{array}{c}\text { LANDSAT } 7 \text { ETM+ } \\
\text { (Path/row: } 014 / 051 ; \text { date: } 26 / 07 / 2001) \\
\end{array}$} \\
\hline & Banda 1 & Banda 2 & Banda 3 & Banda 4 \\
\hline Maximum Deep of Penetration: Zi (m) & 21.4 & 16.8 & 5.2 & 3.0 \\
\hline
\end{tabular}

Fuente: CIAF-DIMAR (estudio propio, 2013).

Un modelo numérico de transferencia radiativa se utilizó para medir y corregir la reflectancia esperada en la parte superior de la columna de agua, dicha medida está derivada de la respuesta espectral de un blanco de referencia sumergido en cada zona DOP del hábitat bentónico con el fin de obtener el coeficiente de atenuación en cada una de las islas (Figura 4).

El estudio espectral contempla un análisis estadístico por reducción de desviación estándar SD en cada línea de regresión (i), analizando la covarianza (i, j) entre bandas para cada una de las zonas DOP (Lyzenga, 1981). Tal y como se indica en la siguiente fórmula:

$$
\left.K_{i} / K_{j}=a+\sqrt{\left(a^{2}\right.}+1\right)
$$

En donde ( $k i / k j)$ es el coeficiente de atenuación, y a es:

$$
a=\frac{\sigma i i-\sigma j j}{2 \sigma i j}
$$


Y donde:

$$
\sigma i j=\overline{X_{i} X_{j}=\left(\overline{X_{i} X_{j}}\right)}
$$

Compensar el efecto de atenuación de la radiación electromagnética que ejerce la columna de agua en zonas tropicales, incrementa significativamente el nivel de exactitud en la clasificación de fondos de arrecifes de coral (Heather y Ellsworth, 2002).

\section{Interpolación y calibración de la profundidad en las zonas DOP}

Entre el máximo de la profundidad de penetración para cada banda y la superficie, suponiendo que el sustrato se mantiene constante, el valor de DN es puramente una función de la profundidad.

De acuerdo con el modelo de transferencia radiativa de doble-flujo, la medida radiancia en el sensor $(\mathrm{Li})$, de cualquier píxel sumergido se puede expresar entonces como (Green, Edwards y Mumby, 2000):

$$
L i=\text { Liœmean }+(\text { Li superficie }- \text { Lomean }) \cdot \mathrm{e}^{-2} k i z
$$

Donde:

$L i \infty$ mean es el valor promedio de píxeles para la banda $i$ sobre aguas profundas, debido a la reflexión de la columna de agua, la superficie y la dispersión de la atmósfera; Li superficie es el valor DN medio del fondo arenoso a nivel del mar; $k i$ es el coeficiente de atenuación para la banda $i$ y $z$ es la profundidad.

Con el fin de linealizar la relación entre radiancia y la profundidad (Figura 4), la radiancia corregida atmosféricamente ( $\mathrm{Li}$ - Liœmean) puede ser transformada usando logaritmos naturales:

$$
\operatorname{Ln}(\text { Li - Liœmean })=\operatorname{Ln}(\text { Li superficie }- \text { Lomean })-2 k i z
$$

Esta ecuación puede ser transformada para interpolar la profundidad $(Z)$, en cada zona DOP:

$$
Z=(A i-X i) / 2 k i
$$

Como:

$$
\begin{gathered}
A i=\operatorname{Ln}(\text { Li superficie }- \text { Lomean }) \\
X i=(L i-L i \infty \text { mean })
\end{gathered}
$$


Jupp (1988) y Green et al. (2000) hicieron las siguientes aproximaciones en caso de no conocer los parámetros de Li superficie y ki de las siguientes zonas DOP:

$$
\begin{gathered}
A i=X i \min +2 k i z \\
K i=(X i \max -X i \text { min }) / 2(Z i-Z i+1)
\end{gathered}
$$

Como:

$$
\begin{aligned}
& X i \min =\operatorname{Ln}(\text { Li min }- \text { Lomean }) \\
& X i \text { max }=\operatorname{Ln}(\text { Li max }- \text { Lomean })
\end{aligned}
$$

Cuando $L i$ min y $L i$ max son, respectivamente, el valor mínimo y máximo de DN en cada zona DOP; $\mathrm{Zi}$ es la profundidad máxima de penetración para la zona DOP $i$ y $Z i+l$ es la máxima profundidad de penetración de la zona superpuesta DOP. Los parámetros necesarios se resumen en la Tabla 3.

Tabla 3

Resumen de los parámetros requeridos para la estimación de la profundidad

\begin{tabular}{lcccc}
\hline & Banda 1 & Banda 2 & Banda 3 & Banda 4 \\
\hline Li media de penetración $(D N)$ & 160 & 134 & 96 & 23 \\
Li max $(D N)$ & 230 & 200 & 130 & 24 \\
Li min $(D N)$ & 90 & 67 & 62 & 21 \\
Zi $(m)$ & 21.4 & 16.8 & 5.2 & 3.0 \\
$X i$ max & 2.07 & 3.25 & 3.93 & 4.38 \\
$X i$ min & 1.79 & 1.60 & 1.79 & 1.09 \\
Ki & 0.03 & 0.07 & 0.48 & 0.54 \\
Ai & 3.13 & 3.99 & 6.85 & 4.38 \\
\hline
\end{tabular}

Fuente: CIAF-DIMAR (estudio propio, 2013).

\section{Resultados y discusión}

La ecuación $(Z=(A i-X i) / 2 k i)$ se aplicó a cada una de las bandas así: a la banda 1 para la zona DOP-1, a la banda 2 para la zona DOP- 2; etcétera. Los resultados separados para cada zona DOP se combinaron en un mapa batimétrico de los arrecifes de coral para la isla de San Andrés, como se puede ver en la Figura 5. 
Las profundidades representadas aquí hacen referencia al datum vertical. La exactitud del mapa resultante está sujeta a la comprobación en campo con el fin de comprobar la fuerza de correlación, expresada por el coeficiente de correlación de Pearson, entre la profundidad obtenida y la profundidad real (Figura 5).

Algunos errores fueron causados por los supuestos o fórmulas inherentes al método utilizado. Como ya se ha mencionado por Ji, Civco y Kennard (1992), la precisión de los resultados se debe, por tanto, más a la aplicación de correcciones radiométricas y atmosféricas de los datos de satélite en la zona de estudio. Desafortunadamente, la información auxiliar sobre las condiciones atmosféricas en el momento de adquisición de la imagen es muy escasa, por lo que es imposible de aplicar un modelo de corrección atmosférica absoluta a la imagen.
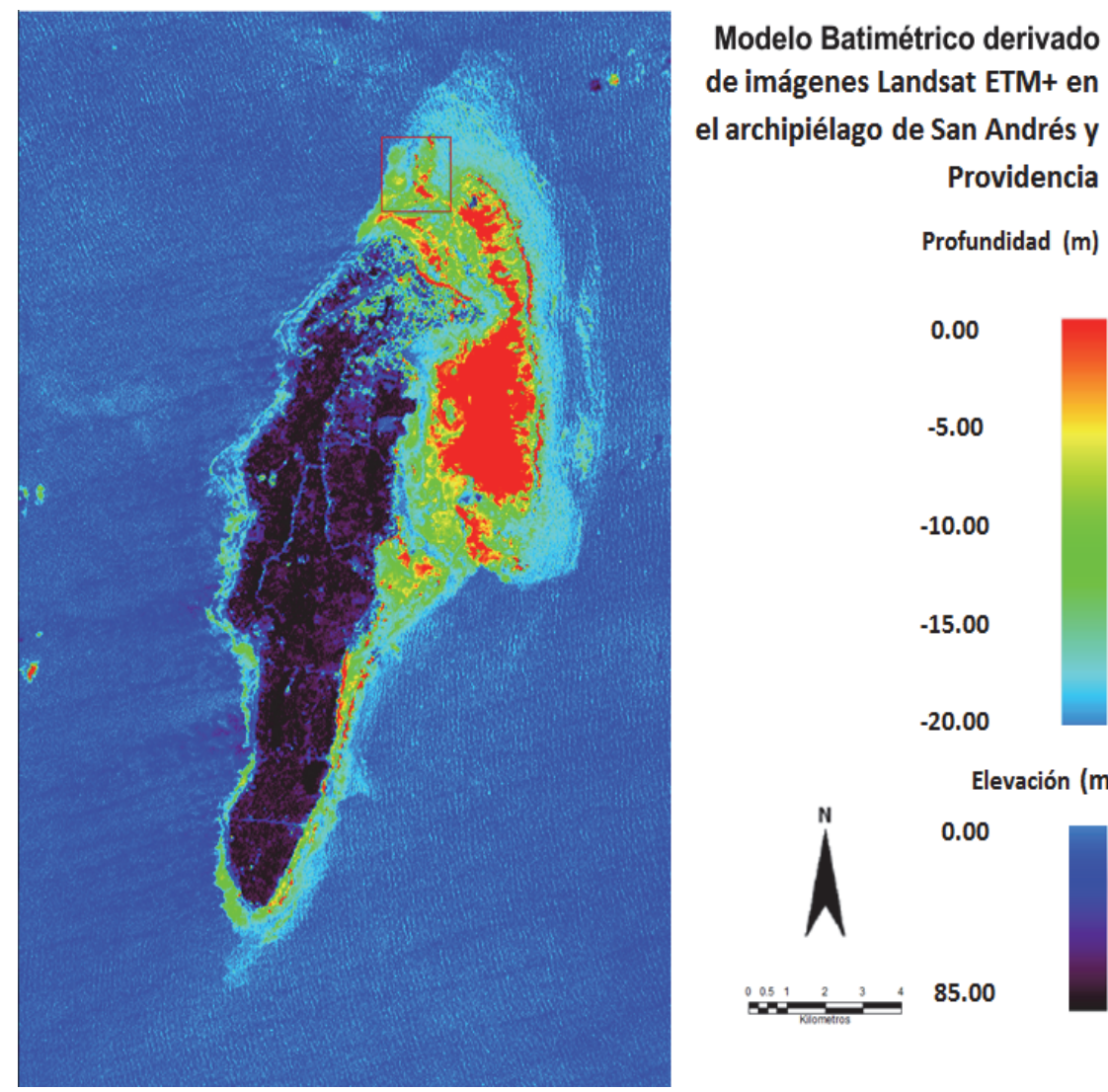

Providencia

Profundidad (m)

0.00

$-5.00$

$-10.00$

$-15.00$

$-20.00$

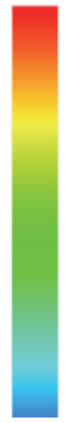

Elevación (m)

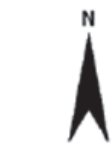

0.00

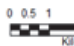

85.00

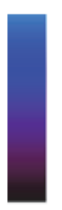

Figura 5. Modelo Batimétrico de las profundidades en la isla de San Andrés (estudio propio, 2013). 


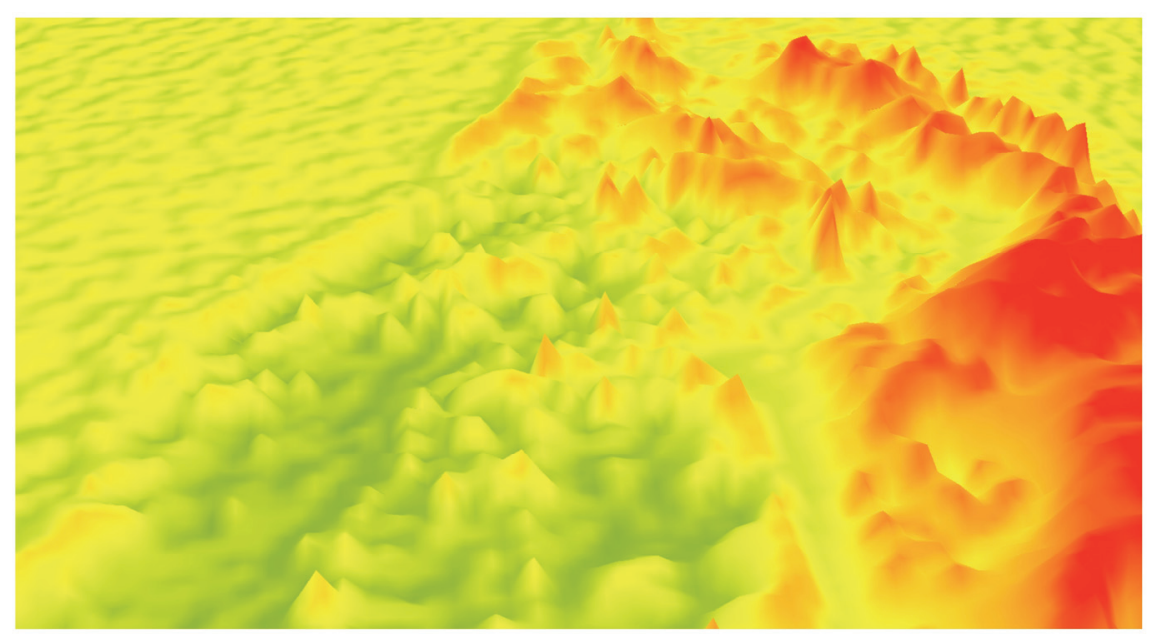

Valores de Batimetria. Noreste isla de San Andrés.

(m)

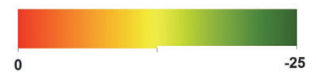

Figura 6. Modelo Batimétrico en 3D generado a partir de la modificación del método DOP, hasta los $-25 \mathrm{~m}$ bajo el nivel del mar en la isa de San Andrés (estudio propio, 2013).

\section{Conclusiones}

Un estudio más profundo en esta área en relación con los modelos batimétricos, facilitaría las posibilidades de desarrollar un sistema de seguimiento de arrecifes de coral en el mar Caribe colombiano basado en percepción remota. Dichas técnicas sirven, en primer lugar, para derivar información acerca de la ubicación de los arrecifes de coral (X, Y coordenadas), la profundidad a la que se producen ( $\mathrm{Z}$ coordenadas, batimetría) (Figura 6) son base en una posible determinación de su estructura (delimitación entre los diferentes tipos de fondo, tales como coral, algas, algas marinas y arena) y su condiciones. A escala regional, los sensores remotos contribuyen en el control de las condiciones ecológicas, física y/o química del archipiélago de San Andrés.

Los modelos de batimetría de los arrecifes de coral derivados mediante sensores remotos tienen las ventajas a la hora de llegar a las zonas que no son fácilmente accesibles por resonancia convencional en embarcaciones, permitiendo el trabajo en grandes áreas de forma sinóptica además de la posibilidad de analizar evolución 
temporal. El método DOP (Profundidad de penetración) utilizado en este trabajo dio resultados razonables de una manera eficiente. Pero como se pudo ver después de la prueba de precisión, existen algunas desviaciones de la realidad sobre el terreno. Lo que implica una necesidad de corrección atmosférica y radiométrica más completa de los datos y de contar con un conjunto suficientemente amplio de verificación.

\section{Bibliografía}

Benny, A. y G. Dawson (1983). "Satellite Imagery as an Aid to Bathymetric Charting in the Red Sea", The Cartographic Journal, pp. 5-16.

Chander, G.; Markham, B.L. y D.L. Helder (2009). "Summary of Current Radiometric Calibration Coefficients for Landsat MSS, TM, ETM+ and EO-1 ALI Sensors”, Remote Sensing of Environment, vol. 113, núm. 5, pp. 893-903.

Clark, P.J. (1997). “Coral Reef Habitat Mapping”, Marine Biology, pp. 193-202.

D., J. (1988). "Background and Extensions to Depth of Penetration (DOP) Mapping Shallow Coastal Waters", Proceedings of the Symposium on Remote Sensing of the Coastal Zone, IV.2.1-IV.2.19.

Edwards, A. (1999). "Applications of Satellite and Airborne Image Data to Coastal Management", Coastal Region and Small Island Papers, UNESCO, París.

Edwards, F. (1987). "Climate and Oceanography", Key Environments: Red Sea, pp. 45-69.

Green, E., Gmumby, P. y A. Edwards (2000). "Mapping Bathymetry in Remote Sensing”, Handbook for Tropical Coastal Management, UNESCO Ed., pp. 219-235.

Heather, H. y LeDrew Ellsworth (2002). "Measuring and Modeling Water Column Effects on Hyperspectral Reflectance in a Coral Reef Environment", Remote Sensing of Environment, pp. 300-308.

Hiroya Yamano, M.T. (2004). "Detection limits of coral reef bleaching by satellite remote sensing", Simulation and Data Analysis. Remote Sensing of Environment, pp. 86-103.

Jerlov, N. (1976). "Marine Optics", Elsevier Oceanographic Series, vol. 14, Elsevier Scientific Publishing Company, Amsterdam.

Ji, W., Civco, D. y W. Kennard (1992). "Satellite Remote Bathymetry", A New Mechanism for Modelling. Photogrammetric Engineering \& Remote Sensing, pp. 545-549.

Lysenga, D.R. (1978). "Passive Remote Sensing Techniques for Mapping Water Depth Anda Bottom Features”, Applied Optics, vol. 17, pp. 379-383.

Mow, J.M. (2005). "Community Visioning Process in Old Providence and Santa Catalina”, Report 1994, UNESCO, Small Islands Voice. 
Nacional, F.D. (2003). Informe final "Modelación del acuífero San Andrés Islas bajo escenarios de cambio climático y usos del recurso, Proyecto INAPColombia", Universidad Nacional, Medellín.

Serge Andréfouët, P.K.-P.-P. (2003). "Multi-site Evaluation of IKONOS Data for Classification of Tropical Coral Reef Environments", Remote Sensing of Environment, pp. 128-143.

U.S. Government Funding (1994). "Manual of Harmonic Analysis and Prediction of Tides", United States Government Printing Office.

Vanderstraete, T.; Goossens, R. y T.K. Ghabour (2003). "Remote Sensing as a Tool for Bathymetric Mapping of Coral Reef in the Red Sea", BELGEO, HurghadaEgypt, pp. 257-269. 